\title{
On Weak Equivalence of Linear Systems and Finite State Systems
}

\author{
A. M. Eshmawi, C. T. Mullis, ${ }^{*}$ and R. A. Roberts* \\ Electrical and Computer Engineering Dept., Faculty of Engineering, \\ King Abdulaziz University, Jeddah, Saudi Arabia.
}

\begin{abstract}
It is shown that finite state machines exist which are weakly equivalent to linear systems for some nontrivial definitions of weak equivalence. Two systems, one linear with state space $R^{n}$, the other with finite state spece, operate on the same stationary uncorrelated input sequence $u$. The two systems have real valued output sequences $y$ and $\hat{y}$. Notions of weak equivalence are formulated, which involve sets of second moments of the input and two outputs. Power spectrum equivalence requires the $E\left(y_{t} y_{t+\tau}\right)=E\left(\hat{y}_{t} \hat{y}_{t+\tau}\right)$ for all $\tau$. Cross-correlation equivalence requires that $E\left(u_{1} y_{t+\tau}\right)=E\left(u_{t} \hat{y}_{t+\tau}\right)$ for all $\tau$. The interdependence of these and other notions of weak equivalence are studied. The existence of weakly equivalent finite state systems is constructively demonstrated for a standard class of linear systems.
\end{abstract}

\section{Introduction}

Let us agree to call the system

$$
\left\{\begin{array}{l}
X_{t+1}=A x_{t}+b u_{t} \\
y_{t}=c x_{t}
\end{array}\right.
$$

where $x_{t} \in R^{n}$, and $u_{t}, y_{t} \in R$ a linear system although it is more properly called a discrete-time, finite dimensional, nontime-varying linear system. The vector $x$, is the state, $u_{t}$ the input, and $y_{t}$ the output. We assume that the system (1) is stable, (i.e., its eigenvalues satisfy $|\lambda| \leq 1$ ), controllable, (i.e., $\rho A^{k} b=0$ for all $k \geq 0$ implies $\rho=0$ ), and observable $\left(c A^{k} x=0 \text { for all } k \geq 0 \text { implies } x=0\right)^{[1,2]}$. The system

\footnotetext{
* Department of Electrical and Computer Engineering, University of Colorado. Boulder, Co 80302.
} 


$$
\left\{\begin{array}{l}
\phi_{t+1}=f\left(\phi_{t}, u_{t}\right) \\
\hat{y}_{t}=g\left(\phi_{t}\right)
\end{array} \quad-\infty<t<\infty\right.
$$

is a finite state system if $\phi_{t} \in \Phi=\{1,2, \ldots, m\}$, i.e., the state space is a finite set even though the output $u_{t}$ and output $\hat{y}_{t}$ are real valued.

We shall consider the question "when are the systems (1) and (2) in some sense equivalent?" The motivation for this question lies in the fact that physical simulations of the ubiquitous linear system are performed on digital machines, which are in reality finite state systems. The approximation is generated by discretizing the linear system, and usually, the discretization error is negligible. This requires however that the number $m$ of elements of $\Phi$ be quite large. There are two questions which occur about such an approximation.

First, how good an approximation can be obtained if $m$ is constrained, i.e., what is the tradeoff between number of states and output error variance $E\left(y_{t}-\hat{y}_{1}\right)^{2}$ ? This question is the more practical one and is considered by Koplowitz ${ }^{[3]}$ and Mullis and Roberts $^{54}$. In fact, the problem is closely related to the classical problem of discretization error in numerical analysis, such as may be found in $\mathrm{Liu}^{[5]}$ and $\mathrm{Max}^{[6]}$.

The second question is more theoretical. What sort of notions of system equivalence can be formulated so that the system (1) and (2) are precisely equivalent? These involve a "white noise" test input, and mixed second moments involving the sequences $\left\{u_{t}\right\},\left\{y_{t}\right\}$ and $\left\{\hat{y}_{t}\right\}$ which are then stationary second-order random processes.

It is obvious that the error $y_{t}-\hat{y}_{t}$ depends on the nature of the common input sequence $\left\{u_{t}\right\}$. Since $\Phi$ is finite, one can choose $\left\{u_{t}\right\}$ to generate an arbitrary error. In order to generate a reasonable problem, therefore, one must choose a reasonable class of inputs. There are three more or less standard test inputs for linear systems; a unit pulse, sinusoids and white noise. The unit pulse response and the frequency response each characterize the input-output relation for a linear systems. However, this is no longer true for nonlinear systems and was proposed by Wiener ${ }^{[7]}$ for this purpose. We shall thus take $\left\{u_{t}\right\}$ to be an independent and identically distributed random sequence with $E\left(u_{t}^{2}\right)=1$ and $E\left(u_{t}\right)=0$. The distribution function of $u_{t}$ is

$$
F(u)=\operatorname{Pr}\left\{u_{t} \leq u\right\}
$$

The cross-correlation sequence $E\left[u_{t} y_{t+\tau}\right]$ of the input and output sequences of (1) is known to be the unit pulse response of the system, under the conditions we have imposed on $\left\{u_{t}\right\}$. This fact provides an identification technique which has in fact been realized in hardware. This suggests a notion of weak system equivalence. We shall call the systems (1) and (2) cross-correlation equivalent if $E\left[u_{t} y_{t+\tau}\right] \equiv E\left[u_{t} \hat{y}_{t+\tau}\right]$.

The autocorrelation sequence $E\left[y_{t} y_{1+\tau}\right]$ determines the power spectrum of the output process. In many situations, such as signal generation, the output power spectrum is the only important property of the system. We shall call the systems (1) and (2) power spectrum equivalent if $E\left[y_{t} y_{t+\tau}\right] \equiv E\left[\hat{y}_{t} \hat{y}_{t+\tau}\right]$. The problem of constructing 
Markov chains with a real valued output having a specified power spectrum has some precedence in the signal processing literature ${ }^{[8,9]}$. In fact constructing digital signal sources, which are widely used in the digital communication field is supposed to be a good practical application for the results of the work in this paper.

We shall constructively demonstrate the existence of cross-correlation and power spectrum equivalent finite state systems in this paper.

\section{Pulse Response and Autocorrelation Sequences}

The common input process $\left\{u_{t}\right\}$ for the systems (1) and (2) is independent and identically distributed. Therefore, since the right hand sides of (1) and (2) do not depend explicitly on time, the state trajectories $\left\{x_{t}\right\}$ and $\left\{\phi_{t}\right\}$ are stationary Markov processes. So in fact, is $\left\{x_{t}, \phi_{t}\right\}$. We shall briefly discuss these processes and consider all mixed second moments involving elements of the input sequence and the output sequences $\left\{y_{t}\right\}$ and $\left\{\hat{u}_{t}\right\}$.

The sequence $\left\{x_{t}\right\}$ is a stationary, second-order, $R^{n}$-valued process with mean

$$
E\left(x_{t}\right)=0
$$

and covariance

$$
E\left[x_{t} x_{i+\tau}^{T}\right]=K\left(A^{\tau}\right)^{T}, \tau \geq 0
$$

Here

$$
K=A K A^{T}+b b^{T}=\sum_{k=0}^{\infty}\left(A^{k} b\right)\left(A^{k} b\right)^{T}
$$

since $(A, b)$ is stable and controllable, the sum in (6) exists and $K$ is positive definite. The scalar output sequence satisfies

$$
y_{t}=c x_{t}=\sum_{\tau=1}^{\infty} h_{\tau} u_{t-\tau}
$$

where

$$
h_{\tau} \triangleq c A^{\tau-1} b=E\left[u_{t} y_{t+\tau}\right], \quad \tau \geq 1
$$

The sequence $\left\{h_{t}\right\}$ is called the pulse response for the linear system. The autocorrelation sequence for the stationary process $\left\{y_{i}\right\}$ is

$$
r_{\tau} \triangleq E\left[y_{t} y_{t+\tau}\right]=c K\left(c A^{\tau}\right)^{T}=\sum_{i=1}^{x} h_{t} h_{t+\tau}
$$

for $\tau \geq 0$.

The sequence $\left\{\phi_{t}\right\}$ is a stationary Markov chain taking values in $\Phi=\{1,2, \ldots, m\}$. The next-state function $f$ and distribution function $F$ determine an $m \times m$ transition matrix $Q$ and an associated $m \times m$ matrix $R$ via

$$
\underline{Q}\left(\phi, \phi^{\prime}\right)=\left.\right|_{\left\{u, f(\phi . u)=\phi^{\prime}\right\}} d F(u)
$$




$$
R\left(\phi, \phi^{\prime}\right)=\int_{\left\{u: f(\phi, u)=\phi^{\prime}\right\}} u d F(u)
$$

(We will usually adhere to the convention that components of vectors and matrices indexed by $\Phi$ be identified by arguments. Subscripts will usually denote time). We have

$$
\begin{gathered}
\operatorname{Pr}\left\{\phi_{t+1}=\phi^{\prime} \mid \phi_{t}=\phi\right\}=Q\left(\phi, \phi^{\prime}\right) \\
E\left\{u_{t} \mid \phi_{t+1}=\phi^{\prime}, \phi_{t}=\phi\right\}=\frac{R\left(\phi, \phi^{\prime}\right)}{Q\left(\phi, \phi^{\prime}\right)}
\end{gathered}
$$

Let $l$ denote the $m$-dimensional column vector whose components are all unity. Then

$$
\begin{aligned}
& Q I=1 \\
& R I=0
\end{aligned}
$$

(since the input is zero-mean). The finite system (2) (or the matrix $Q$ ) is ergodic if for some $t$, every component of $Q^{t}$ is positive. (Note that ergodicity implies a "controllability" property, since for any pair of states in $\Phi$ there will be a trajectory of positive probability which joins them). If $Q$ is ergodic, then $Q^{\prime} \rightarrow I p$ where $p$ is a probability vector. The vector $p$ is the unique solution to $p l=1, p Q=p$ and satisfies

$$
\operatorname{Pr}\left\{\phi_{t}=\phi\right\}=p(\phi)>0 \text { for all } \phi
$$

Let $g$ be the output map in (2); the column vector whose $\phi$-th component is $g(\phi)$. Suppose that the system (2) is ergodic. Then the output process $\left\{\hat{y}_{l}\right\}$ has autocorrelation sequence

$$
\begin{aligned}
\hat{r}_{\tau} & \triangleq E\left[\hat{y}_{t} \hat{y}_{t+\tau}\right] \\
& =E\left[g\left(\phi_{t}\right) g\left(\phi_{t+\tau}\right)\right] \\
& =\sum_{\phi . \phi^{\prime}} p(\phi) Q^{\tau}\left(\phi, \phi^{\prime}\right) g(\phi) g\left(\phi^{\prime}\right) \\
& =g^{T} D_{p} Q^{\tau} g, \quad \tau \geq 0
\end{aligned}
$$

where

$$
D_{p}=\operatorname{diag}\{p(1), \ldots, p(m)\}
$$

(we use this solution throughout. Note that if $Q$ is ergodic, $D_{p}$ is nonsingular).

The state of a deterministic system characterizes an equivalence class of past input sequences, motivating the use of the word "memory". Let us consider the memory (in the mean) that the finite state machine (2) has of its past intput.

$$
\begin{aligned}
& E\left[u_{t-\tau} \mid \phi_{t}\right]=E\left[E\left[u_{t-\tau} \mid \phi_{t-\tau}=\phi, \phi_{t-\tau+1}=\phi^{\prime}, \phi_{l}\right] \mid \phi_{l}\right] \\
& =\sum_{\phi, \phi^{\prime}}\left[\frac{R\left(\phi, \phi^{\prime}\right)}{Q\left(\phi^{\prime}\right)}\right]\left[\frac{p(\phi) Q\left(\phi, \phi^{\prime}\right) Q^{\tau-1}\left(\phi^{\prime}, \phi_{t}\right)}{p\left(\phi_{l}\right)}\right]
\end{aligned}
$$




$$
=\left[p R Q^{\tau-1} D_{p}^{-1}\right]\left(\phi_{t}\right), \quad \tau>0
$$

Using this result, we may compute the cross-correlation of the input and output sequences for the finite state machine. In analogy with the pulse response of the linear system, define for $\tau>0$

$$
\begin{aligned}
\hat{n}_{\tau} & \triangleq E\left[u_{t} \hat{y}_{t+\tau}\right] \\
& =E\left|E\left[u_{t} g\left(\phi_{t+\tau}\right) \mid \phi_{t+\tau}\right]\right| \\
& \left.=\sum_{\phi \in \Phi} p(\phi) g(\phi) \mid p R Q^{\tau-1} D_{p}^{-1}\right](\phi) \\
& =p R Q^{\tau-1} g
\end{aligned}
$$

We shall call the sequence $\left\{\hbar_{\tau}\right\}$ the statistical pulse response for the finite state machine.

One can express the expectation of the product of any two elements drawn from the sequences $\left\{u_{t}\right\},\left\{y_{1}\right\},\left\{\hat{y}_{t}\right\}$ in terms of the sequences $\left\{h_{\tau}\right\},\left\{h_{\tau}\right\},\left\{\hat{r}_{\tau}\right\}$. The sequence $\left\{r_{\tau}\right\}$ is expressible in terms of $\left\{h_{\tau}\right\}$ (equation (9)). The only such expectation that has not been discussed is

$$
\begin{aligned}
E\left[y_{1} \hat{y}_{t+\tau}\right] & =E\left|\left(\sum_{\delta=1}^{\infty} h_{\delta} u_{t-\delta}\right) \hat{y}_{t+\tau}\right| \\
& =\sum_{\delta=1}^{\infty} h_{\delta} h_{\tau+\delta},
\end{aligned}
$$

where $h_{\tau}=0 \quad$ for $\tau \leq 0$.

\section{Weak System Equivalence}

Two systems are generally considered equivalent if they can't be distinguished externally; that is, identical inputs produce identical outputs. We will call the systems (1) and (2) externally equivalent if

$$
E\left(y_{t}-\hat{y}_{t}\right)^{2}=0
$$

It is unreasonable to expect that external equivalence would be possible unless the system (1) is somehow trivial. Therefore it is of interest to consider weaker notions of equivalence. The following are of special interest in the theory of linear systems. We shall call the systems (1) and (2).

(i) cross-correlation equivalent if $h_{\tau}=h_{\tau}$ for all $\tau>0$

(ii) power spectrum equivalent if $r_{\tau}=\hat{r}_{\tau}$ for all $\tau \geq 0$

Each of these notions of equivalence involve the systems (1) and (2) and the distribution function $F$.

One can compute the mean squared error $E\left(y_{t}-\hat{y}_{t}\right)^{2}$ from the pulse response and 
autocorrelation sequences as follows.

Using (9), (17), and (21), we have

$$
\begin{aligned}
E\left(y_{t}-\hat{y}_{t}\right)^{2} & =r_{0}-2 \sum_{\tau-1}^{\infty} h_{\tau} h_{\tau}+\hat{r}_{0} \\
& =\left|\sum_{\tau=1}^{\infty}\left(h_{\tau}-h_{\tau}\right)^{2}\right|=\left|\hat{r}_{0}-\sum_{\tau=1}^{\infty} \hat{h}_{\tau}^{2}\right|
\end{aligned}
$$

The two bracketed error terms in (23) have the following interpretation. Define an "equivalent linear" system for the finite state machine to be the linear system defined by

$$
\tilde{y}_{t}=\sum_{\tau=1}^{\infty} h_{\tau} u_{t-\tau} .
$$

Then the first term in (23), namely

$$
\|h-\hat{n}\|^{2}=E\left(y_{t}-\tilde{y}_{t}\right)^{2}
$$

is the mean squared error between the two linear systems. The second term

$$
\hat{r}_{0}-\|h\|^{2}=E\left(\hat{y}_{t}-\tilde{y}_{t}\right)^{2}
$$

is the mean squared error between the finite state machine and its equivalent linear system. This can serve as measure of nonlinearity, for the finite state system.

For a given next-state map $f$, the output majp $g$ which minimizes $E\left(y_{t}-\hat{y}_{t}\right)^{2}$ must agree with the conditional expectation, i.e.,

$$
\begin{aligned}
g(\phi) & =E\left[y_{t} \mid \phi_{t}=\phi\right] \\
& =\sum_{\tau=1}^{\infty} h_{\tau} E\left[u_{t-\tau} \mid \phi_{t}=\phi\right] \\
& =\sum_{\tau=1}^{\infty} h_{\tau}\left(p R Q^{\tau-1} D_{p}^{-1}\right)(\phi)
\end{aligned}
$$

We will call this map the mininum variance output map. For machines with minimum variance output maps,

$$
\begin{aligned}
\hat{r}_{\tau} & =g^{T} D_{p} Q^{\tau} g \\
& =\sum_{t=1}^{\infty} h_{t}\left(p R Q^{t+\tau-1} g\right) \\
& =\sum_{t=1}^{\infty} h_{t} h_{t+\tau}
\end{aligned}
$$


It follows that $E\left[\hat{y}_{t} \hat{y}_{t+\tau}\right]$ (see (21)).

It the systems (1) and (2) are externally equivalent, then they are cross-correlation equivalent and power spectrum equivalent. Furthermore (24) holds. The following proposition considers the converse.

\section{Proposition 1}

Suppose that the finite state system (2) is ergodic. The following three statements are incompatible in the sense that if any two hold, then the systems (1) and (2) must be externally equivalent.
(a) $h_{\tau}=h_{\tau}, \quad, \tau \geq 0$
(Cross-correlation equivalence)
(b) $\hat{r}_{\tau}=r_{\tau}, \quad, \tau \geq 0$
(Power spectrum equivalence)
(c) $\hat{y}_{t}=E\left(y_{t} \mid \phi_{t}\right)$
(Minimum variance output map)

\section{Proof}

If (c) holds, then (25) holds and for $\tau=0$ we have

$$
\hat{r}_{0}=\sum_{t=1}^{\infty} h_{t} h_{t}
$$

Consequently (23) reduces to

$$
E\left(y_{1}-\hat{y}_{1}\right)^{2}=r_{0}-\hat{r}_{0}
$$

If (a) holds, then (23) reduces to

$$
E\left(y_{t}-\hat{y}_{t}\right)^{2}=\hat{r}_{0}-r_{0} .
$$

If (b) holds, then $r_{0}=\hat{r}_{0}$. Consequently, if any two of the three holds, then $E\left(y_{t}-\hat{y}_{t}\right)^{2}=0$.

Is external equivalence possible? Given the disparity between the state spaces of the systems (1) and (2), it is obvious that if they are externally equivalent, then the linear system must trivialize. The following proposition characterizes this situation.

\section{Proposition 2}

There exists a finite state machine externally equivalent to the system (1) if and only if $\left\{\tau: h_{\tau} \neq 0\right\}$ is finite, and there is a finite set $U$ for which $\operatorname{Pr}\left\{u_{t} \in U\right\}=1$.

Let $U$ be the support of $\operatorname{Pr}\{$. $\}$. Suppose that $n=\max \left\{\tau: h_{\tau} \neq 0\right\}<\infty$ and that $U$ is finite. We may then take $\Phi=U^{n}$ with obvious choices of $f$ and $g$ to construct a finite state machine externally equivalent to the system (1).

On the other hand suppose that $E\left(y_{t}-\hat{y}_{t}\right)^{2}=0$ for some finite state system. Then with probability one, $y_{t}$ can take on only finitely many values. Consider the inputoutput map (7). We must have $h_{k} \neq 0$ for some $k>0$ since $n \geq 1$ and the system (1) is a minimal realization. Assuming $U$ is infinite means that $u_{t-k}$ can take on infinitely many values, leading to a contradiction. Therefore $U$ is finite. But $U$ must contain more than one element since $u_{t}$ has mean zero and variance one. If the set $\left\{\tau: h_{\tau} \neq 0\right\}$ were infinite, then the sequence $\left\{h_{0}, h_{1}, \ldots\right\}$ would contain infinitely many values 
(since $h \in \ell_{2}$ ). Therefore, the set of possible right hand sides of (7) is infinite; again a contradiction. Q.E.D.

External equivalence is possible only in trivial situations. However, we shall show (Section 5) that if $(A, b)$ is stable and controllable and $F($.$) is continuous, then there$ exist finite state machines which are cross-correlation equivalent to (1) and finite state machines which are power spectrum equivalent to (1). The construction of these machines is via an "internal" approximation, wherein the state $x_{t}$ of (1) is estimated, rather than merely the output $y_{i}$.

\section{Internal System Equivalence}

Let $\hat{x}: \Phi \rightarrow R^{n}$ be a map which assigns a point in $R^{n}$ to each element $\phi$ of the state space of (2), and satisfies

$$
c \hat{x}(\phi)=g(\phi) \quad \text { for all } \phi \in \Phi
$$

We will think of $\hat{x}\left(\phi_{t}\right)$ as an "estimate" for $x_{t}$ and will call a machine described by the maps $(f, \hat{x})$ an internal approximation of the system (1). Let $\hat{X}$ be the $n \times m$ martix whose $\phi$-th column is $\hat{x}(\phi)$. Then the vector $g$ with components $\{g(\phi)\}$ is $g=(c \hat{x})^{T}$.

The idea of estimating the state of the linear system suggests further notions of system equivalence. We will now list some of these and partially catalogue the dependence of the weak equivalences in the list. If all hold, then the systems must be internally equivalent, i.e., the covariance of the vector $x_{t}-\hat{x}\left(\phi_{t}\right)$ must vanish. In the following, we assume that system (2) is ergodic.

$$
\begin{gathered}
E\left[\hat{x}\left(\phi_{t}\right)\right]=E\left(x_{t}\right)=0, \quad \text { or } \\
\sum_{\phi \in \phi} p(\phi) \hat{x}(\phi)=\hat{x}_{p}^{T}=0 .
\end{gathered}
$$

$$
\begin{gathered}
E\left[\hat{x}\left(\phi_{t}\right) \hat{x}\left(\phi_{t}\right)^{T}\right]=E\left[x_{t} x_{t}^{T}\right]=K, \quad \text { or } \\
\sum_{\phi \in \Phi} p(\phi) \hat{x}(\phi) \hat{x}(\phi)^{T}=\hat{x} D_{p} \hat{x}^{T}=K
\end{gathered}
$$

$$
\begin{gathered}
\hat{x}\left(\phi_{t}\right)=E\left[x_{t} \mid \phi_{t}\right] \quad \text { (minimum variance condition), or } \\
\hat{x}=A \hat{x}\left(D_{p} Q D_{p}^{-l}\right)+b p R D_{p}^{-l}=\sum_{\tau=0}^{\infty} A^{\top} b p R Q^{\top} D_{p}^{-l} .
\end{gathered}
$$

$$
\begin{gathered}
E\left[\hat{x}\left(\phi_{t+1}\right) \mid \phi_{t}\right]=A \hat{x}\left(\phi_{t}\right), \quad \text { or } \\
A \hat{x}=\hat{x} Q^{T} \\
E\left[u_{t} \hat{x}\left(\phi_{t I 1}\right)\right]=E\left(u_{t} x_{t I 1}\right], \quad \text { or } \\
\hat{x}(p R)^{T}=b
\end{gathered}
$$

\section{Proposition 3}

(i) $E 3 \Rightarrow E I$ 
(ii) $E 4 \Rightarrow E$ I

(iii) $E 4, E 5 \Rightarrow$ internal cross-correlation equivalence, i.e.,

$$
E\left[u_{t} \hat{x}\left(\phi_{t+\tau}\right)\right]=E\left(u_{t} x_{t+\tau}\right), \quad \tau>0
$$

(iv) $E 2, E 4 \Rightarrow$ internal power spectrum equivalence, i.e.,

$$
E\left[\hat{x}\left(\phi_{t}\right) \hat{x}\left(\phi_{t+\tau}\right)^{T}\right]=E\left[x_{t} x_{t+\tau}\right], \quad \tau \geq 0
$$

(v) $E 2, E 3 \Rightarrow$ internal equivalence, i.e.,

$$
E\left\{\left[x_{t}-\hat{x}\left(\phi_{t}\right)\right]\left[x_{t}-\hat{x}\left(\phi_{t}\right)\right]^{T}\right\}=0 .
$$

(iv) $E 3, E 4, E 5 \Rightarrow$ internal equivalence.

\section{Proof}

$$
E\left[\hat{x}\left(\phi_{t}\right)\right]=E[E[x, \mid \phi]]=E\left[x_{t}\right] .
$$

(ii) Take the expectation of $E 4$ to get $(A-I) E\left[\hat{x}\left(\phi_{t}\right)\right]=0$

Since $A$ is stable, 1 is not an eigenvalue of $A$, and $A-I$ is invertible.

$$
\begin{aligned}
E\left[u_{t} x_{t 1,}\right] & =A^{\tau-1} b \\
& =A^{\tau-1} \hat{x}(p R)^{T} \\
& =\hat{x}\left(p R Q^{\tau-1}\right)^{T} \\
& =E\left[u_{1} \hat{x}\left(\phi_{t+\tau}\right)\right] \\
K\left(A^{T}\right)^{\tau}= & \hat{x} D_{p} \hat{x}^{T}\left(A^{T}\right)^{\tau} \\
= & \hat{x} D_{p} Q^{\tau} \hat{x}^{T} \\
= & \sum_{\phi . \phi^{\prime}}\left[p(\phi) Q_{\tau}\left(\phi, \phi^{\prime}\right) \hat{x}(\phi) \hat{x}\left(\phi^{\prime}\right)^{T}\right]
\end{aligned}
$$

(v) Geven E2,

$$
\operatorname{cov}\left(x_{t}-\hat{x}\left(\phi_{t}\right)\right)=K-E\left[x_{t} \hat{x}\left(\phi_{t}\right)^{T}\right]-E\left[\hat{x}\left(\phi_{t}\right) x_{t}^{T}\right]+K
$$

But from $E 3$

$$
\begin{aligned}
E\left[x_{1} \hat{x}\left(\phi_{t}^{\prime}\right)\right] & =E\left[E\left[x_{t} \mid \phi_{t}\right] \hat{x}\left(\phi_{t}\right)^{T}\right] \\
& =E\left[\hat{x}\left(\phi_{t}\right) \hat{x}\left(\phi_{t}\right)^{T}\right] \\
& =K
\end{aligned}
$$

(vi) In veiw of (v) it suffices to show that $E 3, E 4, E 5 \Rightarrow E 2$

Thus

$$
\begin{aligned}
\hat{x} D_{p} \hat{x}^{\prime} & =A \hat{x} D_{p} Q \hat{x}^{\prime}+b p R \hat{x}^{\prime} \\
& =A\left(\hat{x} D_{p} \hat{x}^{I}\right) A^{T}+b b^{\prime}
\end{aligned}
$$

Since $A$ is stable, the solution $K$ to $(6)$ is unique. and therefore. $E 2$ holds. Q.E.D.

\section{Markov Chain Approximation}

In the prededing section certain notions of weak equivalence were introduced. In 
section 5 it is shown that the consideration of these equivalence relations is not futile; for any linear system and suitably nontrivial input there exist finite state machines which are either cross-correlation or power spectrum equivalent. In order to construct such finite state machines, one is led to consider the problem which is posed and solved in the present section.

Consider an ergodic finite Markov chain with state space $\Phi=\{1,2, \ldots, m\}$, transition matrix $Q$ defined on $\Phi \times \Phi$ and trajectories $\left\{\phi_{1}\right\}$. These trajectories satisfy the Markov property and the fundamental conditional probabilities (12). Let $\hat{x}: \phi \rightarrow R^{n}$ be a given mapping from the state of the chain into the state spece of the linear system (1). The vector valued sequece $\left\{\hat{x}\left(\phi_{t}\right)\right\}$ then depends only on the trajectory of the Markov chain.

The problem posed in this section addresses the following question. Can the Markov chain and estimator $\hat{x}$ be constructed so that any sample sequence $\hat{x}\left(\phi_{t}\right)$ is also a possible trajectory of the linear system (1)?

It should be clear that not every vector sequence is such a possible trajectory. There must exist a scalar input sequence $\bar{u}_{t}$ (for every sample sequence $\phi_{t}$ ) for which

$$
\hat{x}\left(\phi_{t+1}\right)=A \hat{x}\left(\phi_{t}\right)+b \tilde{u}_{t} \text { for all } t
$$

In other words, the vector $\hat{x}\left(\phi_{t+1}\right)-A \hat{x}\left(\phi_{t}\right)$ must be a scalar multiple of the vector $b$. If this is the case, then the random sequence $\tilde{u}_{t}$ is uniquely of the determined by (27). This pseudo "input" is actually a function of the trajectory $\left\{\phi_{t}\right\}$. (Therefore, the usual cause and effect relationship of input and state are reversed). For the linear system, the present state $x_{t}$ and input $u_{t}$ are independent random variables. We shall require a weaker version for $\tilde{u}_{t}$, namely

$$
E\left[\tilde{u}_{t} \mid \phi_{t}\right]=0
$$

Finally, in order to avoid trivialities we shall also require that

$$
\sigma^{2} \triangleq E\left(\tilde{u}_{t}^{2}\right)>0
$$

An algebraic version of this problem is as follows:

\section{Problem Statement}

Given $(A, b)$ stable and controllable, find an integer $m$, an ergodic transition matrix $Q$, a state estimator function $\hat{x}$ and a real matrix $v$, for which

$$
\left\{\begin{array}{l}
Q(i, j)[\hat{x}(j)-A \hat{x}(i)-b v(i, j)]=0, \quad 1 \leq i, j \leq m \\
\sum_{j=1}^{m} Q(i, j) \cdot v(i, j)=0, \quad 1 \leq i \leq m \\
\hat{x}(i) \neq 0 \quad \text { for some } i
\end{array}\right.
$$

The correspondence of this problem with the original question is established by setting $\tilde{u}_{t}=v\left(\phi_{t}, \phi_{t+1}\right)$. Note that given $(Q, \hat{x})$ the matrix $v$ is essentially determined by $(30) ; v(k, j)$ is arbitrary if $Q(k, j)=0$. It is perhaps not obvious that (30)-(32) guarantee that 


$$
\sigma^{2}=\sum_{i, j=1}^{m} p(i) Q(i, j)[v(i, j)]^{2}>0
$$

(where $p$ is the stationary measure for $Q$ ). We will demonstrate this below. Consider, for the present, certain properties of solutions.

Let $\hat{X}$ be the $n \times m$ matrix whose $\phi$-th column is $\hat{x}(\phi)$. Let $\tilde{R}$ be the $m \times m$ matrix with components

$$
\tilde{R}(i, j)=Q(i, j) \cdot v(i, j)
$$

in analogy with the matrix $R$ in (11). We may then rewrite (31) as

$$
\tilde{R} l=0
$$

which is analogous to (15). Multiply (30) by $p(i)$ and sum over $i$ to get

$$
\hat{x} D_{p}=A \hat{x} D_{p} Q+b p \tilde{R}
$$

This is analogous to (E3). Now sum (30) over $j$, using (34) to get

$$
A \hat{X}=\hat{X} Q^{T}
$$

which is (E4). Multiply (30) by $p(i) v(i, j)$ and sum over $i, j$ to get

$$
\hat{X}(p \tilde{R})^{T}=\sigma^{2} b
$$

which is similar to $(E 5)$. Define

$$
\hat{K}=\hat{X} D_{p} \hat{X}^{T}=E\left[\hat{x}\left(\phi_{t}\right) \hat{x}\left(\phi_{t}\right)^{T}\right]
$$

Equations (36), (37) and (38) may be combined to get

$$
\hat{K}=A \hat{K} A^{T}+\sigma^{2} b b^{T}
$$

Since the only solution to the homogeneous equation $Y=A Y A^{T}$ is the trivial solution $Y=0,(6)$ and (40) imply that

$$
\hat{K}=\sigma^{2} K
$$

It follows that there can be but two possibilities. Either $\hat{X}=0$ and $\sigma^{2}=0$ and $\hat{K}=0$, or $\hat{X} \neq 0, \sigma^{2}>0$ and $\hat{K}$ is positive definite. Thus if (32) holds, then $\sigma^{2}>0$ as claimed.

The random sequence $\left\{\tilde{u}_{t}\right\}$ is uncorrelated. To see this, write

$$
\begin{aligned}
E\left[\tilde{u}_{t} \tilde{u}_{t+\tau}\right] & =E\left[E\left[\tilde{u}_{t+\tau} \mid \phi_{t}, \phi_{t+1}, \phi_{t+\tau}, \phi_{t+\tau+1}\right]\right] \\
& =p \tilde{R} Q^{\tau-1} \tilde{R} l \\
& =0 \quad \text { for } \quad \tau>0, \quad \text { by (35) }
\end{aligned}
$$

(This is a consequence of the Markov property). Finally, consider the covarience function for $\left\{\hat{x}\left(\phi_{t}\right)\right\}$. Using (37) and (41) we find that

$$
E\left[\hat{x}\left(\phi_{t}\right) \hat{x}\left(\phi_{t+\tau}\right)^{T}=\hat{X} D_{p} Q^{\tau} \hat{X}^{T}\right.
$$




$$
\begin{aligned}
& =\left(\hat{x} D_{p} \hat{X}^{T}\right)\left(A^{T}\right)^{\tau} \\
& =\sigma^{2} K\left(A^{T}\right)^{\top}
\end{aligned}
$$

Thus, any special approximation for which $\sigma^{2}=1$ is (internally) power spectrum equivalent to the system (1).

\section{Proposition 4}

There exists a solution to the Markov chain approximation problem.

The proof of the above proposition is based on constructing the Markov chain approximant given in Appendix I. To follow up the example given next, it is recommended to read Appendix I.

\section{Example}

The construction of $(Q, \hat{x})$ is no doubt grossly inefficient. There may be smaller (in the size of $\phi)$ solutions. As an example, consider first order linear systems $(n=1)$ with $0<A<1$. Let $b=1$. We have $L=Z$. and $A(x)$ is the open interval $(-M, M)$ with $M=1 /(1-A)$. Then $U$ is given by

$$
U= \begin{cases}1, & \text { if } A<1 / 2 \\ A /(1-A) & \text { if } A \geqslant 1 / 2\end{cases}
$$

Some analysis shows that

$$
L_{0}=\{k \in Z,-\ell \leq k \leq \ell\}
$$

where $\ell$ is the integer satisfying

$$
U_{2}-1<\ell \leq U_{2}
$$

For the case $A<1 / 2$, the construction yields the following chain (which is also a solution for $1 / 2 \leq A<1$, even though the construction will produce larger chains for this range of values of $A$ ).

$$
\begin{aligned}
& \left.\hat{X}=[-1,0,1] \quad \text { (the points in } L_{0}\right) \\
& {\left[v_{i j}\right]=\left[\begin{array}{ccc}
A-I & A & ? \\
-1 & 0 & 1 \\
? & -A & 1-A
\end{array}\right][\text { to satisfy }(48)]} \\
& Q=\left[\begin{array}{ccc}
A & 1-A & 0 \\
q & 1-2 q & q \\
0 & 1-A & A
\end{array}\right][0<q<1 / 2]
\end{aligned}
$$

This chain is ergodic with stationary measure

$$
p=[\pi, 1-2 \pi, \pi], \quad \pi=q /(1-A+2 Q)
$$

One may readily check to see that $(30),(31)$, and (32) are satisfied. 


\section{Cross-Correlation and Power Spectrum Equivalence}

In section 4 we constructed a Markov chain $\left\{\phi_{t}\right\}$ with a vector valued output $\left\{\hat{x}\left(\phi_{t}\right)\right\}$ and a scalar valued output $\hat{u}_{t}=v\left(\phi_{t}, \phi_{t+1}\right)$ so as to satisfy (27)-(29). In order to construct an honest finite state system (2) we must reverse the cause and effect relationship between $\left\{\phi_{t}\right\}$ and $\left\{\tilde{u}_{t}\right\}$ and relate $\bar{u}_{t}$ to the actual input $u_{t}$. In other words we must construct functions $f$ and $w$ so that if

$$
\begin{gathered}
\phi_{t+1}=f\left(\phi_{t}, u_{t}\right) \\
\tilde{u}_{t}=w\left(\phi_{t}, u_{t}\right)
\end{gathered}
$$

then (27)-(29) hold. Furthermore we must have

$$
\operatorname{Pr}\left\{\phi_{t+1} \mid \phi_{t}\right\}=Q\left(\phi_{t}, \phi_{t+1}\right)
$$

where $(Q, \hat{x})$ is the solution to the special approximation problem. We will assume that the distribution function $F$ is continuous.

Define the functions $f: \Phi \times R \rightarrow \Phi$ and $w: \Phi \times R \rightarrow \Phi$ as follows. Let $v$ be the matrix which appears in (30). For a given $\phi \epsilon \Phi$, let $\left(\theta_{1}, \ldots, \theta_{m}\right)$ be a permutation of $\Phi$ for which

$$
v\left(\phi, \theta_{1}\right) \leq v\left(\phi, \theta_{2}\right) \leq \ldots \leq v\left(\phi, \theta_{m}\right)
$$

Since $F$ is continuous, we may choose numbers $\left\{\alpha_{0}, \ldots, \alpha_{m+1}\right\}$ satisfying

$$
\left\{\begin{array}{l}
-\infty=\alpha_{0} \leq \alpha_{1} \leq \ldots \leq \alpha_{m} \leq \alpha_{m+1}=x \\
F\left(\alpha_{k}\right)-F\left(\alpha_{k-1}\right)=Q\left(\phi, \theta_{k}\right), 1 \leq k \leq m
\end{array}\right.
$$

Then $f$ and $w$ are such that

$$
\alpha_{k-1}<u \leq \alpha_{k} \Rightarrow\left\{\begin{array}{l}
f(\phi, u)=\theta_{k} \\
w(\phi, u)=v\left(\phi, \theta_{k}\right)
\end{array}\right.
$$

By construction, the Markov chain defined by (49) will satisfy (51), and if $\bar{u}_{t}$ is given by (50), then (27) - (29) will hold. Furthermore

$$
E\left[\bar{u}_{t} u_{t}\right]>0
$$

This positive correlation is due to the agreement in the ordering of the $\left\{\alpha_{k}\right\}$ with the order in (52). In other words $u^{\prime}>u \Rightarrow w\left(\phi, u^{\prime}\right) \geq w(\phi, u)$ with strict inequality on a set of positive probability.

\section{Proposition 5}

If $(A, b)$ is stable and controllable, and $F$ is continuous, then there exists a finite state system which is internally cross-correlation equivalent to the linear system (1).

\section{Proof}

Let $(Q, \hat{x})$ be a solution to the special approximation problem. Construct the maps $f$ and $w$ satisfy (53) for each $\phi$. Let $\bar{u}_{t}$ satisfy (50) where $\left\{\phi_{t}\right\}$ is generated by the finite state machine (49). Let $\eta=E\left[u_{t} \bar{u}_{t}\right]>0$. Define a "scaled" estimator 


$$
\hat{x}^{\prime}(\phi)=\eta^{-1} \hat{x}(\phi)
$$

Since (27) and (28) hold, we have

and

$$
E\left[\hat{x}\left(\phi_{t+1}\right) \mid \phi_{t}\right]=A \hat{x}^{\prime}\left(\phi_{t}\right)
$$

$$
\begin{aligned}
E\left[u_{t} \hat{x}^{\prime}\left(\phi_{t+1}\right)\right] & =\eta^{-1} E\left[u_{t} \hat{x}\left(\phi_{t+1}\right)\right] \\
& =\frac{E\left(u_{t}\left(A \hat{x}\left(\phi_{t}\right)+b \bar{u}_{t}\right)\right]}{E\left[u_{t} \bar{u}_{t}\right]} \\
& =b
\end{aligned}
$$

Therefore, for the internal approximation $\left(f, \hat{x}^{\prime}\right)$, the weak equivalences $(E 4)$ and (E5) hold and by Proposition 3, we have internal cross-correlation equivalence.

We could modify this construction to obtain internal power spectrum equivalence, but it is not really necessary since in this case the input is of no relevance. As we mentioned below equation (43), we need only construct a special approximation for which

$$
\sigma^{2}=E\left[\tilde{u}_{t}^{2}\right]=1
$$

This is easily done by the obvious scaling $\hat{x}^{\prime}(\phi)=\hat{x}(\phi) / \sigma^{2}$ of a given special approximation. Therefore we have the following.

\section{Proposition 6}

If $(A, b)$ is stable and controllable, then there exists a finite state system which is internally power spectrum equivalent to the linear system (1).

We have constructively demonstrated the existence of internal cross-correlation equivalent finite state systems and internal power spectrum equivalent finite state systems when $(A, b)$ is stable and controllable and $F$ is continuous. The existence of cross-correlation or power spectrum equivalent finite state systems follows by simply defining $\hat{y}_{t}=g\left(\phi_{t}\right)=c \hat{x}\left(\phi_{t}\right)$.

\section{Conclusion}

The motivation for considering finite state machines (with real-valued inputs and outputs) that are equivalent (in some sense) to a given linear system arises from the widespread use of digital systems for the simulation of linear systems. Fidelity in this context is usually phrased in terms of quantities such as the output mean square error $E\left(\mathrm{y}_{\mathrm{t}}-\hat{\mathrm{y}}_{\mathrm{t}}\right)^{2}$. This error variance can be zero only for certain trivial cases. A different problem is formulated here. Namely, what kinds of equivalence relations are there for which it is possible that a linear system and a finite state machine can be equivalent?

Two nontrivial equivalence relations have been offered: cross-correlation, and power spectrum equivalence. These relations involve two systems and a white noise input process. It was shown that for any stable linear system, if the distribution function $F$ is continuous, then there are finite state machines which are equivalent to the 
linear system in either sense. (Not simulteneously, however, since Proposition 1 showed that this would imply external equivalence, and Proposition 2 showed that this could happen only in trivial cases).

As a corollary, it follows that any power spectrum obtained by shaping white noise with a finite order linear filter can also be obtained with a finite Markov chain. This has been an unproved conjecture.

\section{References}

[1] Kalman, R. Arbib, M. and Fab, P., Topics in Mathematical Systems Theory, (Chap. 10), McGrawHill, New York (1969).

[2] Friedman, Nathoniel A., Introduction to Ergodic Theory, Van Nostrand Rein-hold Mathematical Studies, No. 29, New York (1969).

[3] Koplowitz, J., Estimation and Approximation with a Finite Statistic, Ph.D. dissertation, Department of Electrical Engineering, University of Colorado, Boulder, Colorado (1973).

[4] Mullis, C.T. and Roberts, R.A., Synthesis of minimum roundoff noise fixed point digital filters, IEEE Trans. Circuits and Systems, CS 23-9 (1976).

[5] Liu, B., Effect of finite word length on the accuracy of digital filters-A review, IEEE Trans. On Circuit Th., CT 18: 670-677 (1971).

[6] Max, J., Quantization for minimum distortion, IRE Trans. on Information Th., CT 13: 7-12 (1960).

[7] Wiener, N., Nonlinear Problems in Random Theory, M.I.T. Press, Cambridge (1958).

[8] Mullis, C.T. and Steiglitz, K., Circulant Markov chains as digital signal sources, IEEE Trans. on Audio and Electroacoustics, AU 20: 246-249 (1972).

[9] Sittler, R., Systems analysis of discrete Markov processes, IRE Trans. on Circuit Th., CT 13: 257-266 (1956)

\section{Appendix 1}

\section{Proof : Construction}

In order to find a special approximation, one must first construct a finite set of vectors $\{\hat{x}(\phi)\}$ such that for each $\phi$ there is a $\phi^{\prime}$ for which $\hat{x}\left(\phi^{\prime}\right)-A \hat{x}(\phi)=b v\left(\phi, \phi^{\prime}\right)$. Furthermore the set of all such $v\left(\phi, \phi^{\prime}\right)$ must contain zero in its convex hull. This set will be constructed using a special basis for $R^{n}$. Given such a set, one may construct a transition matrix $Q$ such that (30) and (31) hold. It then becomes necessary to verify that the transition matrix is ergodic.

Consider the following special basis for $R^{n}$. Let

$$
a(z)=z^{n}+a_{1} z^{n-1}+\ldots a_{n}=\operatorname{det}(z l-A)
$$

and let

$$
\psi_{1}=b, \quad \psi_{k+1}=A \psi_{k}+a_{k} b \text { for } k=1, \ldots, n-1
$$

Since $(A, b)$ is controllable, these vectors are linearly independent. By the CayleyHamilton theorem,

$$
A \psi_{n}+a_{n} b=a(A) b=0 .
$$

Consider the lattice $L$ of vectors whose components with respect to the basis $\left\{\psi_{k}\right\}$ are integer valued, i.e., 


$$
L=\left\{\sum_{k=1}^{n} \zeta_{k} \psi_{k}: \zeta_{k} \in Z, \quad k=1,2, \ldots, n\right\}
$$

If $x \in L$, then

$$
\begin{aligned}
A x+b u & =A \sum_{k=1}^{n} \zeta_{k} \psi_{k}+b u \\
& =\sum_{k=1}^{n} \zeta_{k}\left(A \psi_{k}+a_{k} b\right)+b\left(u-\sum_{k=1}^{n} a_{k} \zeta_{k}\right) \\
& =\left(u-\sum_{k=1}^{n} a_{k} \zeta_{k}\right) \psi_{1}+\sum_{k=2}^{n} \zeta_{k-1} \psi_{k}
\end{aligned}
$$

Therefore, $A x+b u$ is also an element of $L$, provided that

$$
u-\sum_{k=1}^{n} a_{k} \zeta_{k} \quad \text { is an integer }
$$

(note that (I 1) was used in (I 2)).

Denote the set of points reachable from the origin via the system (1) with inputs bounded by 1 , in time $t$ by

$$
\mathscr{A}(t)=\left\{\sum_{k=1}^{1} A^{k-1} b u_{k}, \quad\left|u_{k}\right| \leq 1 \text { for } k=1,2, \ldots, t\right\}
$$

Let

$$
\mathscr{A}(x)=\cup_{t \leq 0} \mathscr{A}(t)
$$

Since the eigenvalues of A satisfy $|\lambda|<1,, \mathscr{A}(x)$ is bounded. Impose the norm

$$
\|x\|_{x}=\max \left\{\left|\zeta_{j}\right|: j=1, \ldots, n\right\} \text { where } x=\sum_{j=1}^{n} \zeta_{i} \psi_{j}
$$

and choose $M$ satisfying $x \in \mathscr{A}(x) \Rightarrow\|x\|_{\mathrm{x}}<M$.

\section{Lemma}

For any $x \in L$ there is a trajectory of the system (1) which begins at $x$, remains always in $L$, and passes through the origin in finite time, with $\left|u_{t}\right| \leq M\left(\left|a_{1}\right|+\ldots\left|a_{n}\right|\right)$

\section{Proof}

If $x_{t}=\Sigma_{j=1}^{n} \zeta_{j t} \psi_{j} \in L$, and $u_{t}-\Sigma_{j=1}^{n} a_{j} \zeta_{j} \in Z$, then $x_{t+1} \in L$. Construct $\left\{u_{l}\right\}$ as follows. If $\left\|x_{t}\right\|_{x}>M$, then choose $u_{t}$ so that $u_{t}-\Sigma_{j=1}^{n} a_{j} \zeta_{j}$ is an integer but $\left|u_{t}\right| \leq 1 / 2$. If $\left\|x_{t}\right\|_{\infty} \leq M$, then set $u_{t}-\Sigma_{j=1}^{n} a_{j} \zeta_{j t}=0$. We will still have

$$
\left|u_{t}\right|=\left|\sum_{j=1}^{n} a_{j} \zeta_{j t}\right| \leq M \sum_{j=1}^{n}\left|a_{j}\right|
$$


We can not have $\left\|x_{t}\right\|_{x}>M$ for all $t$, since for each $t$ we would have $x_{t}=A^{\prime} x+z_{t}$, where $z_{t}=\Sigma_{k+1}^{1} A^{k-1} b u_{k}, \quad\left|u_{k}\right| \leq 1 / 2$. Therefore, since $2 z_{t} \in \mathscr{A}(t)$.

$$
\left\|x_{t}\right\|_{x} \leq\left\|A^{t} x\right\|_{x}+M / 2
$$

But $A^{\prime} x \rightarrow 0$. Thus there exists

$$
\tau=\min \left\{t:\left\|x_{1}\right\|_{x}<M\right\}<\infty
$$

By construction, the $\psi_{1}$ component of $x_{\tau+1}$ will be zero, the $\psi_{1}$ and $\psi_{2}$ components of $x_{\tau+2}$ will be zero, etc., (see (I 2)). In fact $x_{t}=0$ for $t \geq \tau+n$. Q.E.D.

$$
U=\max \left\{1, M \sum_{j=1}^{n}\left|a_{j}\right|\right\}
$$

Define $L_{0}$ to be the set of all lattice points in $L$ which are reachable via a trajectory of (1) which begins at the origin, remains always in $L$, and satisfies $\left|u_{t}\right| \leq U$ for all $t$. The set $L_{0}$ is finite since all permissible trajectories are bounded by $U M$. Let $m$ be the number of points in $L_{0}$ and define $\hat{x}$ so that

$$
\{\hat{x}(1), \ldots, \hat{x}(m)\}=L_{0}
$$

For a given $\phi \epsilon \Phi=\{1,2, \ldots, m\}$ consider the possible solutions $\left(\phi^{\prime}, u\right)$ to the relations

$$
\hat{x}\left(\phi^{\prime}\right)=A \hat{x}(\phi)+b u \epsilon L_{0}, \quad|u| \leq U
$$

Let $J$ be the greatest integer in $U$. By the definition (I 3), $J \leq 1$. By the construction of $L$ there are at least $J$ solutions to (I 5) with $u<0$ and at least $J$ solutions with $u>0$. Define $v\left(\phi, \phi^{\prime}\right)=u$ whenever (I 5) holds, arbitrary otherwise. Let $Q$ be any transition matrix satisfying $Q\left(\phi, \phi^{\prime}\right)>0$ if and only if a solution $\left(\phi^{\prime}, u\right)$ to (I 5) exists, and satisfying (31). This is possible because for each there are at least $2 J$ solutions to (I5) (and thus at least $2 J$ positive elements in the $\phi$-th row of $Q$ ). Furthermore, since the numbers $v\left(\phi, \phi^{\prime}\right)>0$ implies that (I5) holds with $u=v\left(\phi, \phi^{\prime}\right)$, equation (30) holds. Finally (32) must hold trivially since the vector $b$ must be in $L_{0}$ and also therefore one of the $\hat{x}(\phi)$.

Therefore we have constructed a Markov chain with transition matrix $Q$, a state estimator $\hat{x}$ and a matrix $v$ satisfying all the conditions of the problem except possibly ergodicity.

Is $Q$ ergodic? The origin is an element of $\mathrm{L}_{0}$, and we may take $\hat{x}(1)=0$. By construction, $Q(1,1)>0$ since $\left(\phi^{\prime}, u\right)=(1,0)$ is a solution to (I5) with $\phi=1$. Furthermore, by the definition of $L_{0}$ and the fact that $Q\left(\phi, \phi^{\prime}\right)$ is positive whenever a solution to (I5) exists, there must be a path of positive probability from the origin $(\phi=1)$ to any other state $\phi^{\prime}$. But by the lemma, there is also a path of positive probability from $\phi^{\prime}$ back to 1 . Let $T$ be sufficiently large so that for any $\phi^{\prime}$ there are paths of length $\leq T$ from $\phi^{\prime}$ to 1 and 1 to $\phi^{\prime}$ of positive probability. Then for any pair $\left(\phi, \phi^{\prime}\right)$ there is a path of positive probability of length $2 T$ which begins at $\phi$ proceeds to 1 , remains there for a time and then proceeds to $\phi^{\prime}$ at $2 T$. Since every element of $Q^{2 T}$ is positive, $Q$ is ergodic. Thus proposition 4 is proved. Q.E.D. 
حول التكافؤ الضعيف بين النظم الخطية والنظم ذات الحالات المحدودة

$$
\text { عبد المجيد عشهاوي ، كليفورد موليس و ر. روبرتس كائس }
$$

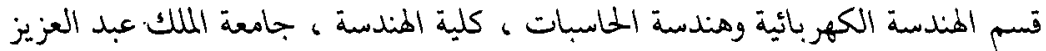

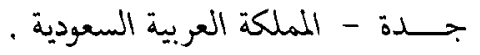

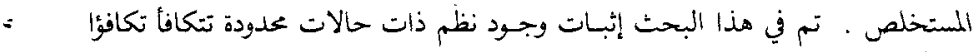

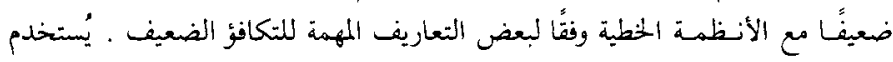

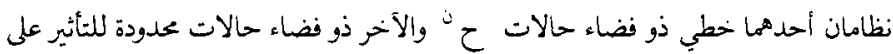

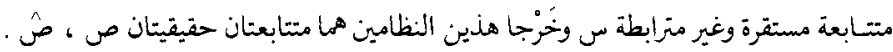

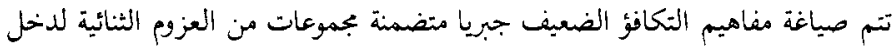

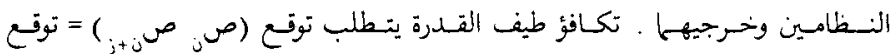

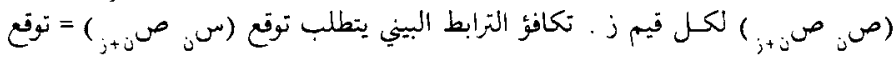

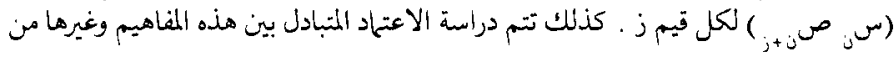

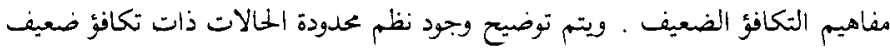
بإنشاء مثال لها لطائفة نمطية من النظم الخطية رئية 